\title{
NATURAL DEDUCTION, INFERENCE, AND CONSISTENCY
}

\author{
ROBERT L. STANLEY
}

1. Introduction. This paper presents results in Quine's elementhood-restricted foundational system $\mathrm{ML},{ }^{1}$ which parallel the important conjecture and theorem which Takeuti developed in his GLC, for type-theoretic systems, and which recur in Schütte's STT. Takeuti conjectured in GLC that his natural deduction subsystem, which does not postulate Modus Ponens or any clearly equivalent inference-rule, is nevertheless ponentially closed. ${ }^{2}$ Further, he showed that if it is closed, then the whole system, including classical arithmetic, must be absolutely consistent.

It is especially easy to establish the "coherence" of suitable, very strong subsystems of ML-that is, that certain (false) formulae cannot be proven under the systems' simple, natural deduction rules. Presumably it is not known whether or not these subsystems are ponentially closed..$^{3}$ If, in fact, they are closed, then they are all equivalent to $\mathrm{ML}$, their coherence implies its coherence, and that in turn implies the consistency of ML and of arithmetic. Proof of such consistency, though much sought, is still lacking. Further, and most important, this inference-consistency relationship suggests the possibility that suitably weakened varieties of these subsystems may be provably consistent, yet usefully strong foundations for mathematics.

2. General argument. ML and other foundational systems for mathematics can be developed by natural deduction, in the same manner as SF. ML conveniently displays the inference-consistency property under examination, which emerges typically in such developments."

Presented to the Society, August 29, 1963; received by the editors February 4, 1964.

1 The abbreviations 'ML', 'GLC', and so on will be used to refer ambiguously to the systems of the articles listed in the bibliography, and to the articles themselves. In any particular case, which sense is meant should be clear from the context.

2 Which is to say, all ponentials of directly provable theorems are themselves directly provable.

${ }^{8}$ Systems can be constructed which are pathologically strong but still ponentially open, and others, which are impractically weak but ponentially closed (see $\$ 4$ below). As for healthy, middle-strength subsystems, the question seems to be open.

${ }^{4}$ Certain minor, artificial changes in the presentation of SF render it amenable to this same line of argument. It is easy to portray ML briefly, however, besides which ML seems to me to be attractively more flexible, powerful, and natural as a foundation for mathematics. 
In SF, Modus Ponens is postulated in effect by the rule FRM. To drop FRM and strengthen the other rules somewhat, ${ }^{5}$ creates a subsystem which is surprisingly strong, considering that it lacks any rules for inferring theorems from other theorems in the usual way. In such subsystems, however, simple formulae occur which typically cannot be proven as theorems. Such a formula and its unprovability are detailed below in $\$ 4$, for an appropriate subsystem of ML.

Since this subsystem of ML includes all theorems which are instances of ML's absolute postulational principles, to postulate ponential closure of the subsystem would generate all theorems of ML. If the subsystem already has the property of being ponentially closed, then its theorems already include all those of ML, its unprovable formulae are unprovable in $\mathrm{ML}$, and therefore $\mathrm{ML}$ must be absolutely consistent by the traditional test that it contains the propositional calculus, Modus Ponens, and formulae which are not theorems.

ML contains classical arithmetic, so if the subsystem is ponentially closed then arithmetic is absolutely consistent. Since proof of arithmetic's consistency would require methods as strong as transfinite induction, such methods would be needed for proof that the subsystem is ponentially closed. Furthermore, even such methods have not yet produced a proof of ML's consistency.

In sum, any proof that Modus Ponens holds in this natural deduction subsystem would have to be of transfinite strength, and would prove correlatively the absolute consistency of ML.

3. The natural deduction systems. When built in SF's style of natural deduction, ML still uses exactly its own same notation, primitives, and formal definitions. Instead of from postulational principles and Modus Ponens, however, theorems evolve individually under rules for drawing particular consequences, where these rules are presented and operate exactly as in SF.

3.1. Rules for ML.

$\mathrm{FR} \downarrow$. If (a proof-line is given in which is enjoined ${ }^{6}$ an occurrence of) $[(\phi \downarrow \psi)]$, then (that proof-line may be copied below the original line, except with the enjoined occurrence replaced by an occurrence of $)\lceil(\sim \phi \cdot \sim \psi)\rceil$; that is, ${ }^{7}$

- See footnote nineteen, page 136, in SF.

- As in SF, a formula which (in abbreviated form) is composed exclusively from occurrences of ', ' ' $v$ ' and various formulae, is called an enjunct of those formulaoccurrences, and they are enjoined in it.

7 "Deduction-bars" are convenient, familiar, and will be used here to express rules. The parenthesized qualifications will still apply implicitly in each usage, and other qualifications will be explicated wherever necessary. 


$$
\frac{(\phi \downarrow \psi)}{(\sim \phi \cdot \sim \psi)}
$$

$\mathrm{FR}() . \frac{\phi(\alpha)}{\phi \frac{\zeta}{\alpha}},{ }^{8}$ for any $\zeta ; \frac{\sim(\alpha) \phi}{\sim \phi \frac{\beta}{\alpha}}$, for any new $\beta$.

$\begin{aligned} \mathrm{FR}=. \frac{\phi}{\phi \frac{\alpha^{\prime}}{\alpha}}, & \left.\text { if }\left[\left(\alpha=\alpha^{\prime}\right)\right] \text { conjoins over } \phi\left(\alpha \in{ }^{9},{ }^{9}\right)\right] .\end{aligned}$

$\mathrm{FR} \in \frac{(\zeta \tilde{\in} \hat{\alpha} \phi)}{\left(\beta_{1-k} \in V \cdot \supset \sim \phi \frac{\zeta}{\alpha}\right)}$, if $\zeta$ is $\mathrm{SR}^{10}$ and has free variables $\beta_{1-k}$.

FRM. $\frac{\phi}{(\phi \cdot(\psi \bigvee \sim \psi))}$, for any proof-line $\phi$, and formula $\psi$.

As in SF, an absurdity is any enjunct which, under some or no distributions of '.' through ' $V$ ', becomes a disjunction of contradictory alternatives - that is, in each alternative are conjoined occurrences of some formula $\phi$ and also $\lceil\sim \phi\rceil$. Finally, the one postulational principle is:

If $\phi$ has no free variables, and $[\sim \phi]$ leads to an absurdity under the FR-rules, then $\phi$ is a theorem.

The main reason for including FRM has been to ensure that Modus Ponens is available, so deleting FRM removes the guarantee that the system is ponentially closed. FRM is also a great convenience, however, for helping to derive several truth-functional "auxiliary" rules, plus numerous "formal" rules, the latter mostly being paired one-to-one with the system's formal definitions. Without these de-

${ }^{8}$ Substitution needs to be characterized for the primitives of ML. Wherever any of the free $\eta$-occurrences in $\phi$ are covered by quantifiers whose variables occur free in $\zeta$, take an alphabetical variant of $\phi$, shifting the bound variables of such quantifiers to new variables (say by alphabetical order) which are not free in $\zeta$. Now $[\phi \zeta / \eta]$ is like this variant of $\phi$ (or like $\phi$ itself if there are no such awkward quantifiers), except for having free occurrences of $\zeta$ wherever the variant (or $\phi$ ) had free $\eta$-occurrences.

- If occurrences of $\phi$ and $\psi$ are linked (under abbreviation) by ' ' only, then the $\phi$-occurrence conjoins over this $\psi$-occurrence and all formula-occurrences which are enjoined in it.

10 'SR' means "stratified and restricted". As ML puts it, a formula is SR when it "is formed from a stratified formula by restricting all bound variables to elements." A term $\zeta$ is SR if $\zeta$ is a variable, or if $\zeta$ is $\left[\hat{\alpha}_{\phi} \mid\right.$ and $\phi$ is SR. 
rived, short-cut rules, any ordinary work within the system would become intolerably lengthy, indeed physically impossible. Some of the most frequently used auxiliary rules are:

$$
\begin{array}{ll}
\text { rc. } & \frac{\phi}{(\phi \cdot \phi)} \quad \text { rid. } \frac{(\phi \bigvee \phi)}{\phi} \\
\text { ric. } \frac{(\phi \cdot \psi)}{\phi} \quad \text { rcc. } \frac{(\phi \cdot \psi)}{(\psi \cdot \phi)} \\
\text { rd. } \frac{(\phi \cdot(\psi \bigvee \chi))}{((\phi \cdot \psi) \bigvee(\phi \cdot \chi))} ; \frac{((\psi \bigvee \chi) \cdot \phi)}{((\psi \cdot \phi) \bigvee(\chi \cdot \phi))} \\
\text { ria. } \frac{(\phi \bigvee \psi)}{\phi}, \text { if } \psi \text { is an absurdity. }
\end{array}
$$

Rules for the defined, truth-functional connectives are exactly the same in ML as in SF. The quantificational rules differ correspondingly as the systems $M L$ and SF differ. $R($ ) for ML is the same as FR( ) above.

$$
\mathrm{R} \exists \cdot \frac{\sim(\exists \alpha) \phi}{\sim \phi \frac{\zeta}{\alpha}}, \text { for any } \zeta ; \frac{(\exists \alpha) \phi}{\phi \frac{\beta}{\alpha}}, \text { for any new } \beta
$$

In both $\mathrm{R}($ ) and $\mathrm{R} \exists, \zeta$ may be any term, not just stratified ones as in SF. The weak instantiation portions of $R($ ) and $R \exists$ are the same in ML and SF. Most of the other rules listed in SF undergo change, as their ML-correspondents show.

$$
\begin{array}{ll}
\mathrm{R}=. & \frac{(\zeta=\eta)}{(\theta \in \zeta \cdot \theta \in \eta \cdot \bigvee \cdot \theta \tilde{\in} \zeta \cdot \theta \tilde{\in} \eta)}, \text { for any } \theta \\
& \frac{(\zeta \neq \eta)}{(\alpha \in \zeta \cdot \alpha \tilde{E} \eta \cdot \bigvee \cdot \alpha \tilde{\in} \zeta \cdot \alpha \in \eta)}, \text { for any new } \alpha . \\
\mathrm{RS}={ }^{\prime} . \quad & \frac{\phi}{\phi \frac{\zeta}{\alpha}}, \text { if }\lceil(\alpha=\zeta)\rceil \text { or }\lceil(\zeta=\alpha)\rceil \text { conjoins over } \phi, \text { and } \phi \text { is either } \\
\mathrm{RS}=. \quad \frac{\phi}{\phi \frac{\zeta}{\alpha}}, \text { if }[(\zeta=\eta)\rceil \text { conjoins over } \phi .
\end{array}
$$


$\mathrm{R} \in . \quad \frac{(\zeta \in \hat{\alpha} \phi)}{\left(\zeta \in V \cdot \phi \frac{\zeta}{\alpha}\right)} ; \frac{(\zeta \tilde{\in} \hat{\alpha} \phi)}{\left(\zeta \tilde{\in} V \cdot V \cdot \sim \phi \frac{\zeta}{\alpha}\right)}$.

$\mathrm{R} C$. (analogous to $\mathrm{R}=$ ).

$\mathrm{R}^{\prime} . \quad \frac{(\zeta \tilde{\in} \iota \zeta)}{(\zeta \tilde{\in} V)}$.

R;. $\quad \frac{\left(\zeta ; \eta=\zeta^{\prime} ; \eta^{\prime}\right)}{\left(\zeta=\zeta^{\prime} \cdot \eta=\eta^{\prime}\right)}, \begin{aligned} & \text { if }\lceil(\zeta \in V)],\lceil(\eta \in V)] \text { and }\left[\left(\eta^{\prime} \in V\right)\right] \text { conjoin over } \\ & \text { the given formula-occurrence; }\end{aligned}$

$$
\frac{\left(\zeta ; \eta \neq \zeta^{\prime} ; \eta^{\prime}\right)}{\left(\zeta \neq \zeta^{\prime} \cdot \vee \cdot \eta \neq \eta^{\prime}\right)}
$$

$\mathrm{R}^{\wedge} \cdot \frac{\hat{\alpha} \hat{\beta} \phi(\zeta, \eta)}{\left(\zeta, \eta \in V \cdot \phi \frac{\zeta, \eta}{\alpha, \beta}\right)} ; \frac{\sim \hat{\alpha} \hat{\beta} \phi(\zeta, \eta)}{\left(\sim(\zeta, \eta \in V) \bigvee \sim \phi \frac{\zeta, \eta}{\alpha, \beta}\right)}$.

$\mathrm{R}^{6} . \quad \frac{\left(\zeta \in \lambda_{\alpha} \eta^{6} \theta\right)}{\left(\zeta \in V \cdot \zeta \in \eta \frac{\theta}{\alpha}\right)}$, if $[(\theta \in V)]$ and $\left[\left(\eta \frac{\theta}{\alpha} \in V\right)\right]$ conjoin

$\frac{\left(\zeta \tilde{\in} \lambda_{\alpha} \eta^{6} \theta\right)}{\left(\zeta \tilde{\in} V \cdot V . \zeta \tilde{\in} \eta \frac{\theta}{\alpha}\right)}$, if $\lceil(\theta \in V)\rceil$ and $\left[\left(\eta \frac{\theta}{\alpha} \in V\right)\right]$ con-

$\mathrm{R} \lambda . \quad \frac{\lambda_{\alpha} \zeta(\eta, \theta)}{\left(\eta, \theta \in V \cdot \eta=\zeta \frac{\theta}{\alpha}\right)} ; \frac{\sim \lambda_{\alpha} \zeta(\eta, \theta)}{\left(\sim(\eta, \theta \in V) \bigvee \sim\left(\eta=\zeta \frac{\theta}{\alpha}\right)\right)}$.

3.2. The subsystem ML'. When FRM is withdrawn from the set of foundation rules, not all of the derived rules still hold. In general, an infinite variety of subsystems is suggested then, corresponding to the infinitely many subsets of derived rules which could be selected as foundation rules for a subsystem. Only one specimen will be developed here; the careful investigation of extended spectra of such subsystems appears important, but probably very long-range. For the present subsystem, ML', the postulated rules will be: FR $\downarrow$, $\mathrm{FR}\left(\mathrm{)}, \mathrm{RS}={ }^{\prime}, \mathrm{FR} \in \mathrm{R} \iota^{\prime},{ }^{11} \mathrm{rc}\right.$ and $\mathrm{rd}$. From this set, proofs of theo-

${ }^{11}$ I believe that this rule does not need to be given foundational status. Conjunction of $\left[\left(\zeta \tilde{\in}_{\llcorner\zeta}\right)\right]$ with $[(\zeta \in V)],[(\zeta \in \eta)]$, or any other such positive elementhoodimplying clause leads to absurdity, thereby satisfying all anticipated proof-needs. I have been unable to derive $[(\zeta \tilde{\in} V)]$ from $\{(\zeta \tilde{E} \iota \zeta)]$ under the $M L^{\prime}$-rules alone, 
rems appear to go through as smoothly as before. Of course, whether all proofs in ML can be supplanted by proofs in $\mathrm{ML}^{\prime}$ remains the crucial, open question-to know that the answer is affirmative would be to know that ML, and arithmetic, are absolutely consistent.

4. Coherence of $\mathrm{ML}^{\prime}$. It is quite easy to show that $\mathrm{ML}^{\prime}$ is coherent - that is, that there exist formulae of $M L^{\prime}$ which cannot possibly have proofs in $\mathrm{ML}^{\prime}$. One such formula is ' $(x)(x \in x)$ '.

In general, here, quantifications and membership formulae will be called even, $[(\phi \downarrow \psi)]$ will be odd just in case $\phi$ and $\psi$ are both even, and $[(\phi \downarrow \psi)]$ even when $\phi$ and $\psi$ are odd. Many formulae are neither even nor odd, as $[(\phi \supset \phi)]$, and $[(\phi \cdot \sim \phi)]$. $\lceil\sim \phi\rceil$ is $[(\phi \downarrow \phi)]$, and thus is odd when $\phi$ is even, even when $\phi$ is odd. Any enjunctive context proves to be even, so that an enjunct as a whole is even if and only if all its enjoined formulae are even, and is odd just when they are all odd.

Any proof of ' $(x)(x \in x)$ ' must commence with its denial, ' $\sim(x)(x \in x)$ '. As the denial of a quantification, this line, as a whole, is odd.

FR $\downarrow$, rc, and rd can be applied to odd formulae, but they cannot act on a given odd formula and yield an enjoined even formula. If $\lceil(\phi \downarrow \psi)]$ is odd (or even), then FR $\downarrow$ gives $[(\sim \phi \cdot \sim \psi)]$, which will be odd (or even) correspondingly; if $\phi$ is odd (or even), so is its result $[(\phi \cdot \phi)]$ under rc; and similarly for rd. Thus oddness and evenness are "invariant" under these three rules, and, in fact, under all valid truth-functional rules. Now an odd occurrence $[\sim(\alpha) \phi]$ can indeed give an even result

$$
\left\lceil\sim \phi \frac{\beta}{\alpha}\right\rceil
$$

under the weak half of $\mathrm{FR}()$, in case $\phi$ is itself odd. In the proof-line ' $\sim(x)(x \in x)$,' however, ' $(x \in x)$ ' is even, so the application of $\mathrm{FR}($ ) could only yield the odd result ' $(x \tilde{\in} x)$ '.

The other foundation rules, $\mathrm{FR} \in, \mathrm{R} \iota$ ', $\mathrm{RS}=$ ', and the strong half of FR( ) cannot operate in the absence of enjoined, even, formulaoccurrences. $\mathrm{FR} \in$ and $\mathrm{R} \iota^{\prime}$ both need an enjoined occurrence of the form $\lceil(\zeta \tilde{\in} \hat{\alpha} \phi)\rceil$, which is defined by $[\sim(\exists \beta)(\zeta \in \beta .(\alpha)(\alpha \in \beta . \supset \phi))]$ if $\zeta$ is a variable, or else by $[\sim(\exists \beta)(\beta=\zeta \cdot \beta \in \hat{\alpha} \phi)]$ if $\zeta$ is an abstract. In either case the prefix $\lceil\sim(\exists \beta)]$ expands into $\lceil\sim \sim(\beta) \sim\rceil$, so that however, when $\zeta$ is an arbitrary abstract. Since $[(\zeta \tilde{\in} \iota \zeta)]$ arises frequently, whereas $\lceil(\zeta \tilde{\in} V)]$ is the preferred elementhood form in the whole development, $\mathrm{R}^{\prime}$ will be taken as initial here, even though its irredundancy is uncertain. 
the whole formula is even. For $\mathrm{RS}={ }^{\prime}$, either $[(\alpha=\zeta)]$ or $[(\zeta=\alpha)]$ must be available. Under the definition of ' $=$ ', an enjoined quantification, and therefore an enjoined even formula, is involved in either case. Similarly for the strong portion of FR( ), which requires precisely an enjoined occurrence of a quantification.

Since the given proof-line is odd, the only rules which can be applied to it are FR $\downarrow, \mathrm{rc}, \mathrm{rd},{ }^{12}$ and the weak portion of FR( ). Since these rules preserve oddness in this application, all enjoined parts must continue odd in the next line, and similarly through all further lines. Every derivable line is, perforce, an odd enjunct.

In order that any enjunct be absurd, occurrences of some $\phi$ and $[\sim \phi]$ must be enjoined in it. If any such $\phi$ is odd, then $[\sim \phi\rceil$ is even, and vice versa. In every line which can evolve from ' $\sim(x)(x \in x)$ ', however, every enjoined formula is odd, and none is even. Therefore no absurdity can be reached below ' $\sim(x)(x \in x)$ ', under the rules of ML', and therefore, finally, $\mathrm{ML}^{\prime}$ contains formulae such as ' $(x)(x \in x)$ ' which cannot be proven in $\mathrm{ML}^{\prime}$.

It is suggestive to drop the $\mathrm{SR}$-restriction in FRE. This modification creates an inconsistent system, with, for example, ' $(R \in R \cdot R \tilde{\in} R$ )' being provable, where ' $R$ ' abbreviates ' $\hat{x}(x \tilde{\in} x)$ '. Nothing changes, however, in the proof-status of ' $(x)(x \in x)$ ', so it remains unprovable. Consequently, this inconsistent variation of ML clearly cannot be ponentially closed, or else all formulae, including ' $(x)(x \in x)$ ', would be provable from the contradictions, via Modus Ponens and the propositional calculus. Oppositely, if all rules except FR $\downarrow$ are dropped, still enough of $M L^{\prime}$ remains to give exactly the propositional calculus, which is ponentially closed. $\mathrm{ML}^{\prime}$ lies somewhere between these two poles. Conceivably it may be both consistent and ponentially closed, or perhaps consistent but ponentially open, lacking some subset of the theorems of ML. At the worst, of course, it might even be inconsistent yet ponentially open-both too strong (in theorems) and too weak (in inference).

A very important line of investigation is the study of various strong systems which, as was suggested in $\$ 1$, are weaker than ML'. Specifically, useful subsystems for which Takeuti's conjecture is actually false are especially interesting, because proof of such systems' consistency would not entail the consistency of the full, ponentially closed systems, hence might be attainable by elementary means. The

12 rd can enter, via steps as follow: $\sim \phi,(\phi \downarrow \phi)$ (by D1), $(\sim \phi \cdot \sim \phi) \quad(\mathrm{FR} \downarrow)$, $(\sim \phi \cdot(\phi \downarrow \phi))(\mathrm{D} 1),\left(\sim_{\phi} \cdot(\sim \phi \cdot \sim \phi)\right)(\mathrm{FR} \downarrow),\left(\sim \phi \cdot\left(\sim \sim_{\phi} \downarrow \sim \sim \phi\right)\right)(\mathrm{D} 2),(\sim \phi \cdot \sim$ $(\sim \sim \phi))(\mathrm{D} 1),(\sim \phi \cdot \sim(\sim \phi \downarrow \sim \phi))(\mathrm{D} 1),\left(\sim \phi \cdot\left(\sim \phi \bigvee \sim_{\phi}\right)\right)(\mathrm{D} 3),\left(\sim \phi \cdot \sim_{\phi} \vee \sim \sim_{\phi}\right.$ $\sim \phi$ ) (rd). D1, D2, and D3 are as in ML. 
question whether any particular subsystem is ponentially open or ponentially closed is certainly important, but the open systems are more promising and interesting than the closed, as candidates for consistency investigation.

5. Summary comment. Methods of natural deduction are shifting more and more of the strength needed for proving theorems, strength traditionally drawn from the powerful rules for mediate inference, into the provisions for direct, self-contained demonstrations of individual theorems. In the natural deduction systems discussed here, this shift has led incidentally to a surprising result. Although the regular system has become hard to distinguish in theorem-content from its strong, "noninferential" subsystem, the two regions turn out to be separated by a serious gulf when one considers the possibility that they are identical-that the system and subsystem actually may have the same body of theorems. Crucially involving consistency, this gulf demands exploration as a possible boundary between bodies of theorems which may prove to be consistent yet "strong enough" for heavy mathematical use, and the traditional, inferentially closed bodies which are too strong for acceptable consistencyproof. Such exploration reaches considerably beyond the limits of the present discussion.

\section{BIBLIOGRAPHY}

GLC. G. Takeuti, On a generalized logic calculus, Japan. J. Math. 23 (1953), 39-96. ML. W. V. Quine, Mathematical logic, rev. ed., Harvard Univ. Press, Cambridge, Mass., 1951.

SF. R. L. Stanley, Simplified foundations for mathematical logic, J. Symbolic Logic 20 (1955), 123-139.

STT. K. Schütte, Syntactical and semantical properties of simple type theory, J. Symbolic Logic 25 (1960), 305-326.

Portland State College 